\title{
ACTITUDES HACIA LA CIENCIA EN ESTUDIANTES UNIVERSITARIOS DE CIENCIAS
}

\author{
ESPINOSA-GARCÍA, J. ${ }^{1}$ y ROMÁN GALÁN, T. ${ }^{2}$ \\ 'Departamento de Química Física. Facultad de Ciencias. Universidad de Extremadura. 06071 Badajoz. \\ ${ }^{2}$ Instituto de Bachillerato «Pedro De Valdivia». Villanueva de la Serena. Badajoz.
}

\section{SUMMARY}

We have performed a longitudinal study among university students, from eighteen to twenty-three years old, and their attitudes toward Science. This attitude does not become neither linear nor more negative through successive years. In contrast, we have observed a «saw tooth» profile, which is clearer when the number of courses analysed increases and the sex variable is taken into account. In general, the maximums of one sex are coincidental with the minimums of the other.

\section{INTRODUCCIÓN}

Las actitudes hacia la Ciencia, o más precisamente, hacia el aprendizaje de las Ciencias, es un tema fundamental en la investigación educativa, pues junto con el componente cognitivo, entre otros factores, ayudan a comprender el proceso de aprendizaje. Los numerosos estudios realizados en otros países (ver, por ejemplo, Ia excelente revisión bibliográfica de Soler LLopis, 1986) contrastan con los escasísimos resultados obtenidos sobre la población española (Serrano 1986, 1988, Arana et al. 1987). En un trabajo anterior (Espinosa y Romám 1991) contribuimos a este conocimiento con un estudio sobre una población de $2^{\circ}$ de BUP. Propusimos una original «escala de puntuaciones» para cuantificar los resultados de las actitudes hacia la Ciencia, encontrando que, aproximadamente, un $20 \%$ de estos alumnos presentaba una actitud negativa.

Un aspecto interesante es la evolución longitudinal de las actitudes con los cursos estudiados. Existe una coincidencia generalizada de que, cuantos más años de Ciencias cursan nuestros alumnos, menos les gusta, incluso en estudiantes de Ciencias (Head et al. 1985, James y Smith 1985, Simpson y Oliver 1985, Yager y Penick 1986, Serrano 1988). Sin embargo, resulta importante saber si estos resultados son válidos en todos los niveles de enseñanza y si son transportables a nuestro medio cultural, pues la coincidencia con los resultados del trabajo de Serrano (1988) no es concluyente, dado que dos cursos ( $5^{2}$ y $8^{8}$ de EGB) no aportan datos suficientes y pueden enmascarar una situación intermedia no medida.

Siguiendo la línea de investigación sobre actitudes hacia el aprendizaje de las Ciencias emprendido en nuestro grupo, hemos abordado un estudio longitudinal que abarca desde la última etapa de EGB hasta la universidad, donde analizamos la variable sexo y factores como teforma, formación profesional o elección de la carrera en primera opción. Pensamos que es el estudio más / amplio realizado en nuestro país sobre el tema de las actitudes hacia la Ciencia. Los resultados obtenidos en los estudios universitarios son los presentados en este trabajo.

\section{DISEÑO DE LA EXPERIENCIA: OBJETIVOS Y MUESTRA}

Como instrumento para conocer la actitud de los alumnos hacia la Ciencia, hemos utilizado el cuestionario elaborado en un trabajo anterior (Espinosa y Román 1991). En él se plantean diez cuestiones contrarias que el alumno debe puntuar de 0 a 10 (Anexo I). Este cuestio- 
nario se ha mostrado sumamente útil por la facilidad y rapidez para ser contestado en clase, así como por el baremo de puntuaciones (entre 0 y 10 ), bastante próximo a las costumbres del alumno.

La encuesta fue realizada a 125 alumnos de la Facultad de Ciencias (Químicas) y a 115 alumnos de las Escuelas de Formación del Profesorado de Badajoz (63 alumnos) y Cáceres (52 alumnos), de la Universidad de Extremadura'. El reducido número de alumnos encuestados es un reflejo de las tasas de matriculación de estos centros. La encuesta fue realizada durante el primer trimestre del curso 1991-92.

Cuatro son, fundamentalmente, los objetivos del trabajo:

a) Constatar la afirmación generalizada de que, a medida que nuestros alumnos cursan más años de Ciencias, menos les gusta; o de otra manera, constatar si la variación lineal de las actitudes hacia la Ciencia encontrada por algunos autores se mantenía en todos los niveles de la enseñanza.

\section{Figura 1}

Tanto por ciento de alumnos con distintas puntuactones (ordenadas) frente a los distintos cursos de Química. hombres ..... mujeres _._t total; $x 2^{\text {a }}$ BUP.

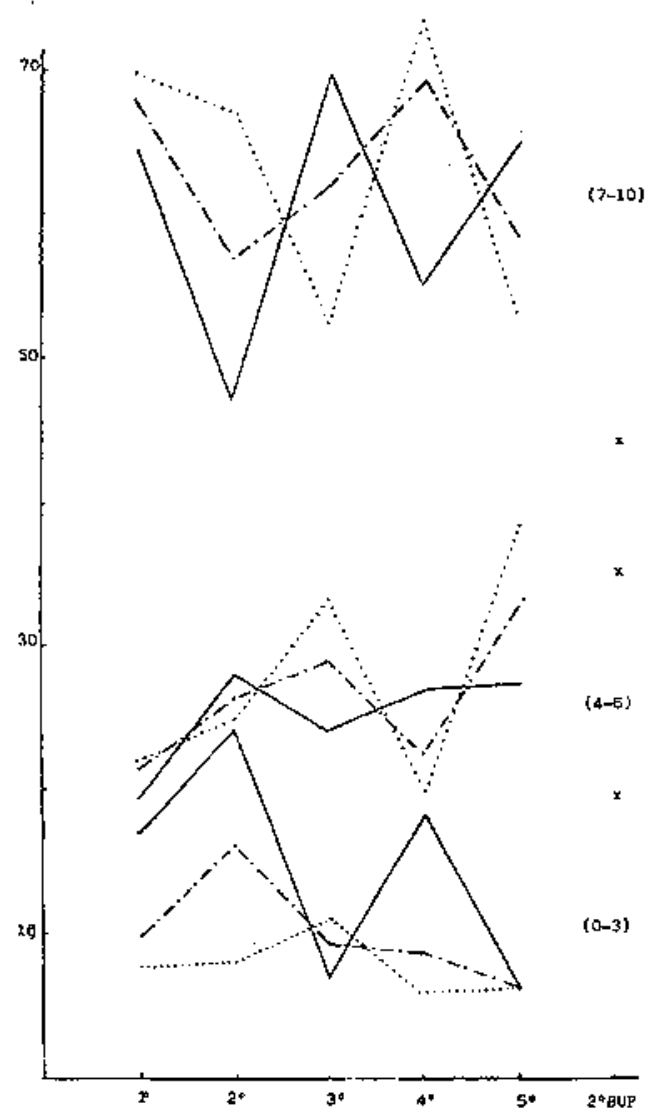

b) Poner de manifiesto la evolución de la actitud hacia la Ciencia en dos carreras universitarias como Magisterio y Químicas, que nos sirviera como una proyección de futuro, pues un alto porcentaje de estos alumnos se incorporarán como profesores de primaria y secundaria.

c) Analizar si la variable sexo es significativa.

d) Comprobar si existe correlación entre la actitud y la elección de la carrera en primera opción.

\section{RESULTADOS Y DISCUSIÓN}

\section{A. Estudios de Química}

Con el objeto de cuantificar los resultados obtenidos, usamos la «escala de puntuaciones» propuesta en otro trabajo (Espinosa y Román 1991). Esta escala se mostró sumamente interesante por su simplicidad y eficacia. Así, agrupamos en cada pregunta el número de alumnos con puntuación 0-3 (actitud negativa), 4-6 (indiferente) y 7-10 (actitud positiva). Los resultados, expresados en \%, aparecen recogidos en la figura 1 , junto con los valores de $2^{\circ}$ de BUP, a título comparativo.

A medida que avanza la licenciatura de Químicas, observamos un perfil en «diente de sierra». Este comportamiento contrasta claramente con los resultados obtenidos en estudios previos de niveles inferiores de la enseñanza, según los cuales, a medida que se avanza en los estudios, la actitud de los alumnos hacia la Ciencia se hace linealmente más negativa ${ }^{2}$. En general, los máximos de un sexo coinciden con los mínimos del otro. La actitud es más positiva que en $2^{\circ}$ de BUP $(57.68 \%$ frente a $46 \%$ ), aunque aún queda un $10 \%$ de alumnos con una actitud negativa hacia la Ciencia. Esta valoración negativa es practicamente el doble en los hombres $(15,1 \%)$ que en las mujeres $(8,3 \%)$. Esta actitud negativa general puede venir motivada por varios factores, entre los que la no elección de la carrera en primera opción puede contribuir en cierta medida, aunque pensamos que no es un factor crucial, pues como veremos más adelante, los estudios de Magisterio presentan un menor nivel de elección en primera opción y, sin embargo, presentan una actitud más positiva.

Por otra parte, cae fuera de los objetivos de este trabajo justificar el comportamiento por sexos, aunque abre las puertas para estudios psicológicos sobrela distinta evolución de la actitud hacia la Ciencia en varones y hembras.

\section{B. Estudios de Magisterio}

Al tratarse solamente de tres cursos, los efectos de la variación de la actitud de los alumnos hacia la Ciencia con el paso de los cursos serán menos nítidos que en Químicas. 
Como en el caso anterior, en la figura 2 aparecen los resultados de la «escala de puntuaciones» (en \%), por sexos, junto con la media total ponderada y los resultados de $2^{\circ}$ de BUP, a título comparativo.

\section{Figura 2}

Tanto por ciento de alumnos con distintas puntuaciones (ordenadas) frente a los distintos cursos de Magisterio.

hombres ..... mujeres _+- total; $x 2^{\circ}$ BUP.

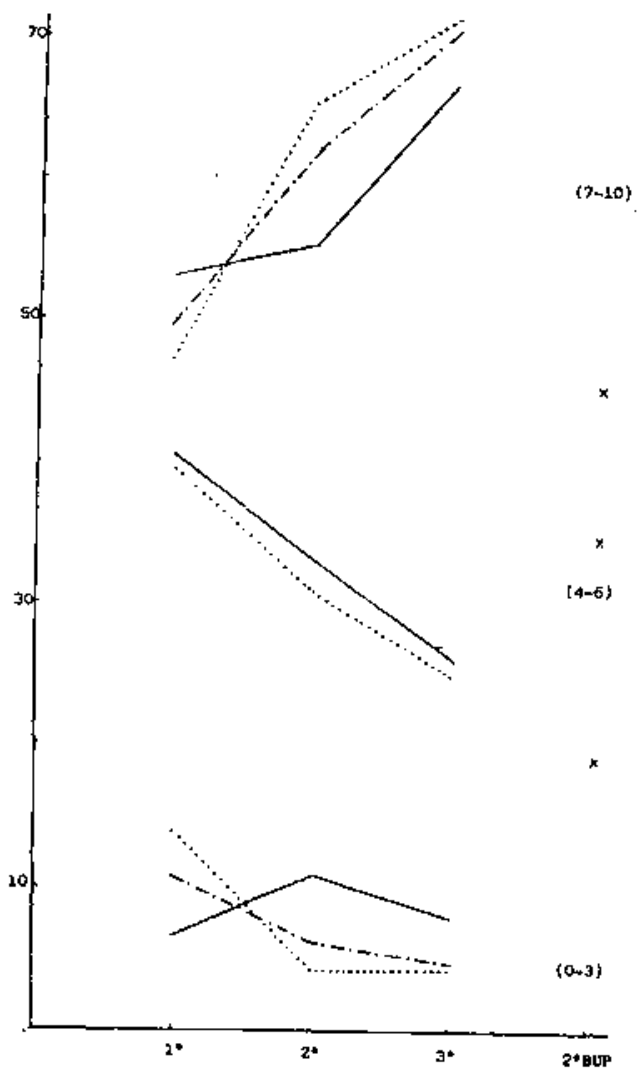

Precisamente por ser sólo tres cursos, el perfil en «diente de sierra" se ve difuminado, especialmente en el caso de los valores medios ponderados. Por otra parte, a medida que avanzan los estudios, disminuye la actitud negativa hacia la Ciencia y aumenta espectacularmente la actitud positiva. Como en Químicas, la valoración negativa es prácticamente el doble en los hombres $(8,0 \%)$ que en las mujeres $(4,2 \%)$.

Englobando la experiencia sobre los dos estudios universitarios analizados (Químicas y Magisterio), finalizamos este trabajo con tres cuestiones. En primer lugar, el bajo porcentaje de actitud negativa hacia la Ciencia en ambos estudios universitarios es un resultado esperanzador, en sí mismo y porque permite suponer que trasladarán esta actitud positiva a sus alumnos cuando ejerzan como futuros profesores. Por otra parte, un tema interesante era conocer si existía alguna correlación entre las actitudes hacia la Ciencia y la elección de la carrera en primera opción. En la tabla I aparecen, expresados en \%, los alumnos con actitud positiva (puntuación 7-10) y negativa (puntuación 0-3), frente a los alumnos de primera opción. No hemos encontrado una correlación entre estas magnitudes, pues en Magisterio tan sólo el $39 \%$, de promedio, de los alumnos la eligen en primera opción (frente al $79 \%$ de la licenciatura de Químicas) y, sin embargo, la actitud positiva hacia la Ciencia es pareja en ambas carreras: $60,3 \%$ en Magisterio frente al $62,8 \%$ en Químicas.

Por último, queremos Ilamar la atención sobre una cuestión metodológica en la investigación sobre actitudes: el número de cursos analizados en una experiencia dada. Así, muchas investigaciones sobre actitudes se basan en los resultados de 2 o 3 cursos para concluir una variación dada de esta actitud con el paso de los cursos. Parece obvio que dos cursos consecutivos no aportan datos suficientes, pues la tendencia siempre será lineal. Sì los dos cursos no son consecutivos, seguirá mostrando una tendencia lineal, pero, además, enmascarando una situación intermedia no medida. En el caso de tres cursos, la situación mejora sustancialmente, pero la variación de la actitud con el paso de los años no va a ser tan nítida como si analizásemos un mayor número de cursos. Por tanto, a la hora de estudiar la variación con los cursos de la actitud hacia la Ciencia ( $y$, en general, cualquier estudio longitudinal sobre una magnitud) seria recomendable usar un número de cursos igual o, preferiblemente, superior a tres.

Tabla I

Actitudes de los alumnos frente a la elección de la carrera en primera opción.

QUÍMICAS

\begin{tabular}{lrrrrrrrrr}
\hline & 1 & 2 & 3 & 4 & 5 & 1 & 2 & 3 \\
\hline ACTITUDES POSITIVAS (7-10) & 68,4 & 57,0 & 61,7 & 68,8 & 58,2 & 49,0 & 61,6 & 70,2 & \\
ACTITUDES NEGATIVAS (0-3) & 9,7 & 16,5 & 9,3 & 8,7 & 8,5 & 11,3 & 6,6 & 4,8 & \\
ALUMNOS EN 19 OPCION & 63,4 & 85,0 & 90,3 & 87,5 & 84,0 & 49,0 & 15,1 & 31,0 & \\
\hline
\end{tabular}

Todos los valores en $\%$ 


\section{CONCLUSIONES}

En primer lugar, a medida que avanzan los cursos en los estudios universitarios analizados, observamos una evolución de la actitud hacia la Ciencia en «diente de sierra». Esta tendencia es más acusada en la licenciatura de Químicas, al tratarse de cinco cursos y si consideramos la variable sexo. Este resultado contrasta claramente con la tendencia generalmente aceptada hasta ahora de evolución lineal y negativa basada en niveles inferiores de la enseñanza.

Por otra parte, en la carreras de Magisterio y Químicas hemos observado una actitud bastante positiva hacia la Ciencia (y mayor que en $2^{\circ}$ de BUP), hecho que nos permite ser optimistas en la proyección de estos alumnos como futuros profesores.

En general, la variable sexo está relacionada con la actitud hacia la Ciencia; las mujeres tienen una actitud menos negativa.

Por último, no hemos encontrado una correlación entre las actitudes hacia la Ciencia y la elección de la carrera en primera opcion.

\section{AGRADECIMIENTOS}

Expresamos nuestro agradecimiento a todos los profesores de la Facultad de Ciencias (Químicas) y las Escuelas de Formación del Profesorado de Badajoz y Cáceres, de la Universidad de Extremadura, que han cooperado pasando las encuestas en sus clases.

\section{NOTAS}

${ }^{1}$ Dado el período de cambio en los planes de estudio en estas escuelas, $1^{\underline{D}}$ de carrera corresponde a la especialidad de primaria, mientras que $2^{\circ}$ y $3^{\circ}$ corresponden a la especialidad de Ciencias. No obstante, como la encuesta fue pasada al principio de curso, los resultados de $1^{\circ}$ recogen la actitud de la experiencia acumulada durante BUP y COU

\begin{abstract}
${ }^{2}$ Para no hacer excesivamente extenso este trabajo, no hemos incorporado las variaciones de la actitud para cada una de las diez cuestiones contrarias planteadas en el Anexo I. En casi todas ellas hemos detectado el perfil en «diente de sierra». Estos resultados están a disposición de toda persona interesada que lo solicite.
\end{abstract}

ENCUESTA DE CUESTIONES CONTRARIAS PUNTUADAS DE 10 A 0

\author{
INTERESANTE / ABURRIDO \\ CLARO / LIOSO \\ FACIL/DIFICIL \\ ME HA ACERCADO A LA NATURALEZA / !NI EN BROMA! \\ PROBARIA OTRA VEZ/ IAMÁS \\ ME HA COSTADO / DEMASIADO FÁCIL \\ HE APRENDIDO FÍSICA Y QUÍMICA / NADA \\ ME HA GUSTADO / NADA \\ ME HE DIVERTIDO / HE SUFRIDO \\ VALÍA LA PENA / TIEMPO PERDIDO
}

\section{REFERENCIAS BIBLIOGRÁFICAS}

ARANA, J., ESCUDERO, T., GARCES, R. Y PALACIAN, E., 1987. Imagen de las asignaturas de Ciencias en la transición de la educación básica a la secundaria, Enseñanza de las Ciencias, 5 (1), pp. 10-15.

ESPINOSA, J. y ROMAN, T., 1991. Actitudes hacia la Ciencia y asignaturas pendientes: dos factores que afectan al rendimiento en Ciencias, Enseñanza de las Ciencias, 9 (2), pp. 151-154.

HEAD, J., LYTH, M. y MAY, J., 1985. The Nuffield 11 to 13 science scheme, School Science Review, 67, 239 , pp. 392-396.

JAMES, R.K. y SMITH, S., 1985. Alienation of students from science in grades 4-12, Science Education, 69 (1), pp. 39-45.

SERRANO, T., 1986. «La imagen de los científicos en los alumnos al finalizar el ciclo medion. Documentos IEPS, monografía 1.
SERRANO, T., 1988. Actitudes de los alumnos y aprendizaje de las Ciencias. Un estudio longitudinal, Investig. Escuela, 5 , pp. 29-38.

SIMPSON, R.D. y OLIVER, J.S., 1985. Attitude toward science and achievement motivation profiles of male and female science students in grades six through ten, Science Education, 69 (4), pp. 511-526.

SOLER LLOPIS, J.B., 1986. Enseñanza de las Ciencias, 4 (2), pp. 174-175.

YAGER, R.E. y PENICK, J.E., 1986. Perceptions of four age group towards science classes, teachers and the value of science, Science Education, 70 (4), pp. 355-364. 